JOURNAL OF

FUNCTION SPACES AND APPLICATIONS

Volume 9, Number 1 (2011), 17-40 (c) 2011, Scientific Horizon

http://www.jfsa.net

\title{
Multiscale homogenization of a class of nonlinear equations with applications in lubrication theory and applications
}

\author{
Andreas Almqvist, Emmanuel Kwame Essel, John Fabricius and \\ Peter Wall
}

(Communicated by Björn Birnir)

2000 Mathematics Subject Classification. 35J60, 35J65, 35B27, 35Q35, $76 \mathrm{D} 08$.

Keywords and phrases. Homogenization; monotone operators; multiscale convergence; two-scale convergence; hydrodynamic lubrication; Reynolds equation; surface roughness; $p$-Laplacian.

Abstract. We prove a homogenization result for monotone operators by using the method of multiscale convergence. More precisely, we study the asymptotic behavior as $\varepsilon \rightarrow 0$ of the solutions $u_{\varepsilon}$ of the nonlinear equation

$$
\operatorname{div} a_{\varepsilon}\left(x, \nabla u_{\varepsilon}\right)=\operatorname{div} b_{\varepsilon},
$$

where both $a_{\varepsilon}$ and $b_{\varepsilon}$ oscillate rapidly on several microscopic scales and $a_{\varepsilon}$ satisfies certain continuity, monotonicity and boundedness conditions. This kind of problem has applications in hydrodynamic thin film lubrication where the bounding surfaces have roughness on several length scales. The homogenization result is obtained by extending the multiscale convergence method to the setting of Sobolev spaces $W_{0}^{1, p}(\Omega)$, where $1<p<\infty$. In particular we give new proofs of some fundamental theorems concerning this convergence that were first obtained by Allaire and Briane for the case $p=2$. 


\section{Introduction}

We study the asymptotic behavior of the solutions $u_{\varepsilon}$ of the nonlinear boundary value problem

$$
\begin{aligned}
\operatorname{div} a_{\varepsilon}\left(x, \nabla u_{\varepsilon}\right) & =\operatorname{div} b_{\varepsilon} & & \text { in } \Omega \\
u_{\varepsilon} & =0 & & \text { on } \partial \Omega
\end{aligned}
$$

as the parameter $\varepsilon$ tends to zero. Here $\Omega$ is assumed to be an open bounded subset of $\mathbb{R}^{N}$ and the functions $a_{\varepsilon}$ and $b_{\varepsilon}$ are assumed to have oscillations in the frequency range of "fast" to "ultra fast", corresponding to the wavelengths $\varepsilon, \varepsilon^{2}, \ldots, \varepsilon^{k}$, where $k$ is an arbitrary positive integer. The idea of homogenization is that the effects of the oscillations on the solution is averaged out.

As an application we show that for particular choices of $a_{\varepsilon}$ and $b_{\varepsilon}$ it is possible to analyze the effects of multiscale surface roughness in some interesting linear and non-linear lubrication models. For example the stationary incompressible Reynolds equation, governing the pressure build-up in a fluid film bearing, falls into this category. The surface micro topography is recognized as an important factor in hydrodynamic lubrication. For very thin films, even small roughness becomes significant and the distance between the two surfaces becomes a rapidly oscillating function. In reality, all technical surfaces are rough due to flaws in the manufacturing process - in fact almost smooth surfaces are very expensive to produce - so surface roughness is something modern technology has to deal with. But there is another incentive to understand the influence of surface roughness in hydrodynamic lubrication. It has been observed that smoothening of the surfaces in a fluid film bearing may lead to a decrease in the hydrodynamic performance. Hence, artificially machined roughness or surface texture can also be considered as a parameter in bearing design.

The effects of surface roughness in various lubrication regimes have been studied with homogenization techniques in numerous works, e.g. $[6,7,8,9,10,14,22]$. In these investigations the roughness is assumed to be periodic of a single wavelength $\varepsilon$. The main result of this paper makes it possible to study the effects of multiscale surface roughness with several wavelengths, in some linear and nonlinear lubrication models. In the linear case, the homogenized equation obtained by multiscale convergence coincides with that of [4], which was obtained by the asymptotic expansion method. Thus the present analysis gives a rigorous justification of the latter method.

Multiscale homogenization of the present class of monotone operators has also been studied in [21] by the periodic unfolding method and in $[15,16,18]$ 
by a combination of methods. To our knowledge, the present analysis is the only treatment that relies on multiscale convergence alone. We also give generalizations and new proofs of some fundamental theorems concerning this convergence that were first obtained by Allaire and Briane [2].

\section{Preliminaries and notation}

Throughout this paper, the letters $p, q, \alpha, \beta$ and $\theta$ denote constants that satisfy $1<p<\infty, \frac{1}{p}+\frac{1}{q}=1,0<\theta<1,0<\alpha \leq \min \{1, p-$ $1\}, \max \{2, p\} \leq \beta<\infty$.

A function $f: \mathbb{R}^{N} \rightarrow \mathbb{R}^{N}$ is said to belong to the class $\mathcal{M}_{\alpha, \beta}^{p}(\theta)$ provided the following conditions are satisfied for any $\xi, \eta \in \mathbb{R}^{N}$ :

$$
\begin{aligned}
f(0) & =0, \\
|f(\xi)-f(\eta)| & \leq \theta^{-1}(1+|\xi|+|\eta|)^{p-1-\alpha}|\xi-\eta|^{\alpha}, \\
{[f(\xi)-f(\eta)] \cdot(\xi-\eta) } & \geq \theta \frac{|\xi-\eta|^{\beta}}{(1+|\xi|+|\eta|)^{\beta-p}} .
\end{aligned}
$$

The symbol $\square$ denotes the $N$-dimensional torus $\mathbb{R}^{N} / \mathbb{Z}^{N}$. In most situations, we identify a function defined on $\square$ with its natural extension to a 1-periodic (in each argument) function defined on $\mathbb{R}^{N}$. The subscript 'per' is employed as a reminder of this. Let $k$ be a positive integer. A typical element of the set $\Omega \times \square^{k}$ is denoted by $\bar{x}^{k}=\left(x, y^{1}, \ldots, y^{k}\right)$. If $f$ is a measurable function on $\Omega \times \square^{k}$ and $1 \leq j \leq k$ we denote by $\bar{f}^{j}$ the average of $f$ w.r.t. the last $j$ variables, i.e. $\bar{f}^{j}$ is the function on $\Omega \times \square^{k-j}$ defined by

$$
\bar{f}^{j}\left(\bar{x}^{k-j}\right)=\int_{\square j} f\left(\bar{x}^{k}\right) d y^{k-j+1} \cdots d y^{k} .
$$

By convention we set $\bar{f}^{0}=f$. The function $a_{\varepsilon}: \Omega \times \mathbb{R}^{N} \rightarrow \mathbb{R}^{N}$ appearing in (1) is assumed to be of the form

$$
a_{\varepsilon}(x, \xi)=a\left(x, \frac{x}{\varepsilon}, \ldots, \frac{x}{\varepsilon^{k}}, \xi\right) \quad(x, \xi) \in \Omega \times \mathbb{R}^{N}
$$

where $a: \Omega \times \square^{k} \rightarrow \mathcal{M}_{\alpha, \beta}^{p}(\theta) \subset C\left(\mathbb{R}^{N} ; \mathbb{R}^{N}\right)$ is a function of Carathéodory type. The function $b_{\varepsilon}$ is of the form

$$
b_{\varepsilon}(x)=b\left(x, \frac{x}{\varepsilon}, \ldots, \frac{x}{\varepsilon^{k}}\right), \quad \text { where } b \in L^{q}\left(\Omega ; C_{\mathrm{per}}\left(\square^{k}\right)\right)^{N} .
$$

The dual of a Banach space $X$ is denoted by $X^{\prime}$. Moreover, for $x \in X$ and $f \in X^{\prime},\langle f, x\rangle$ denotes the value of the linear functional $f$ at the 
point $x$. The Banach space $W_{\text {per }}^{1, p}(\square)$ consists of periodic functions that have weak derivates (in the sense of distributions) and finite Sobolev norm. The subscript ' $\sharp$ ' on a function space indicates that it consists of periodic functions with zero integral, e.g. $f$ is said to belong to $L_{\sharp}^{p}(\square)$ provided $f \in L_{\text {per }}^{p}(\square)$ and $\int_{\square} f d x=0$. By analogy to the notational convention $W^{-1, q}(\Omega)=W_{0}^{1, p}(\Omega)^{\prime}$ we write $W^{-1, q}(\square)=W_{\text {per }}^{1, p}(\square)^{\prime}$. Moreover, $W_{\sharp}^{-1, q}(\square)$ consists of linear functionals $f$ in $W^{-1, q}(\square)$ such that $\langle f, 1\rangle=0$.

A weak solution of $(1)$ is defined as an element $u_{\varepsilon}$ of $W_{0}^{1, p}(\Omega)$ satisfying

$$
\int_{\Omega} a_{\varepsilon}\left(x, \nabla u_{\varepsilon}\right) \cdot \nabla \phi d x=\int_{\Omega} b_{\varepsilon} \cdot \nabla \phi d x
$$

for all $\phi \in W_{0}^{1, p}(\Omega)$. Let $\mathcal{A}_{\varepsilon}: W_{0}^{1, p}(\Omega) \rightarrow W^{-1, q}(\Omega)$ be defined by

$$
\left\langle\mathcal{A}_{\varepsilon}(u), \phi\right\rangle=\int_{\Omega} a_{\varepsilon}(x, \nabla u) \cdot \nabla \phi d x \quad u, \phi \in W_{0}^{1, p}(\Omega) .
$$

By (2b) and Hölder's inequality, we obtain

$$
\left\|\mathcal{A}_{\varepsilon}(u)-\mathcal{A}_{\varepsilon}(v)\right\|_{W^{-1, q}(\Omega)} \leq \theta^{-1}\|1+|\nabla u|+\mid \nabla v\|_{L^{p}(\Omega)}^{p-1-\alpha}\|u-v\|_{W_{0}^{1, p}(\Omega)}^{\alpha} .
$$

Hence $\mathcal{A}_{\varepsilon}$ is continuous. Furthermore, (2c) implies that $\mathcal{A}_{\varepsilon}$ is strictly monotone, i.e.

$$
\left\langle\mathcal{A}_{\varepsilon}(u)-\mathcal{A}_{\varepsilon}(v), u-v\right\rangle \geq 0
$$

with equality if and only if $u=v$. Utilizing (2c) and standard estimates, e.g. Hölder's and Friedrichs' inequality, we can find a positive constant $C$ such that

$$
\left\langle\mathcal{A}_{\varepsilon}(u), u\right\rangle=\int_{\Omega} a_{\varepsilon}(x, \nabla u) \cdot \nabla u d x \geq C\|u\|_{W_{0}^{1, p}(\Omega)}^{p},
$$

provided $\|u\|_{W_{0}^{1, p}(\Omega)}$ is large enough, hence $\mathcal{A}_{\varepsilon}$ is coercive. Throughout the paper the letter $C$ is reserved for a positive constant that may depend on $p, \alpha, \beta, \theta$ or $\Omega$. The value of $C$ may differ from one place to another. Owing to (5), (6) and (7), it is clear that $\mathcal{A}_{\varepsilon}$ satisfies the hypotheses of the Browder-Minty theorem (see e.g. [23] p. 557). Hence, for each $\varepsilon>0$, there exists a unique $u_{\varepsilon}$ that solves (4). Moreover, putting $\phi=u_{\varepsilon}$ in (4) and utilizing (7) and Hölder's inequality we obtain

$$
\left\|u_{\varepsilon}\right\|_{W_{0}^{1, p}(\Omega)}^{p} \leq C\left(1+\left\|b_{\varepsilon}\right\|_{L^{q}(\Omega)}^{q}\right) .
$$


In view of the Riemann-Lebesgue lemma, the sequence $b_{\varepsilon}$ is weakly convergent. Hence it is bounded and we conclude from (8) that the sequence $u_{\varepsilon}$ is bounded in $W_{0}^{1, p}(\Omega)$.

\section{Multiscale convergence}

In 1989 Nguetseng [19] introduced a generalized notion of weak convergence that proved suitable for analyzing homogenization problems with periodic coefficients and one microscopic scale. This technique was later further developed by Allaire [1] who named it two-scale convergence. Two-scale convergence in the setting of Lebesgue spaces $L^{p}(\Omega)$ with $1<p<$ $\infty$ is described in [20]. The benefit of the two-scale convergence technique is that it inherently copes with many of the classical difficulties encountered in homogenization, such as passing to the limit in the product of two weakly convergent sequences.

Homogenization with $k$ microscopic scales, where $k>1$, was initially, as described in the book [11], referred to as reiterated (or iterated) homogenization. In the present context however, we think it is more appropriate to use the term multiscale homogenization. Two-scale convergence (one microscopic scale) was generalized to $(k+1)$-scale convergence or multiscale convergence ( $k$ microscopic scales) in 1996 by Allaire and Briane [2]. As pointed out by these authors (see Remark 2.9 following Theorem 2.6), the case $k>1$ is more delicate due to the possible interactions of the small scales. The results of [2] being valid for $L^{2}(\Omega)$ and $W_{0}^{1,2}(\Omega)$, it has hitherto not been clear whether the multiscale theory extends to $L^{p}(\Omega)$ and $W_{0}^{1, p}(\Omega)$ for all $p$ such that $1<p<\infty$. Nevertheless, many authors seem to have taken for granted that such a generalization is possible, see e.g. Theorem 3.8 in [5] or Theorem 1.7 in [12]. To our knowledge, proofs for $p \neq 2$ have been lacking until now. In this paper we develop a multiscale convergence theory for an arbitrary number of scales and $1<p<\infty$. In particular we give a new proof of Theorem 1.2 in [2] which is preceded by the comment "... its proof is the most difficult (if not important) result of this paper." Another proof of this theorem, also for $p=2$, is due to Balder [3] and relies on the fact that rotation-free vector fields on $Y=(0,1)^{N}$ (the "cell of periodicity") can be represented as gradients of functions in $W_{0}^{1,2}(Y)$. Our proof relies on a different kind of representation (see Theorem 3.3) and is more similar in spirit to that of $[2,19]$.

The cornerstones of the multiscale convergence theory are three theorems related to

(1) compactness of bounded sequences in $L^{p}(\Omega)$, 
(2) multiscale limits of gradients (of bounded sequences in $W_{0}^{1, p}(\Omega)$ ),

(3) multiscale convergence and monotonicity.

With these theorems in place, homogenization of problem (4) becomes a rather short story. In the definition of multiscale convergence stated below the small scales are assumed to be naturally separated, i.e. they are all integer powers of a small parameter $\varepsilon$ : e.g. $\left\{\varepsilon^{j}\right\}_{j=1}^{k}$. In the more general situtation (separated scales), we regard the small scales $\varepsilon_{1}, \ldots, \varepsilon_{k}$ as functions of the parameter $\varepsilon$, that satisfy

$$
\lim _{\varepsilon \rightarrow 0} \varepsilon_{j}=0 \quad \text { for } 1 \leq j \leq k \quad \text { and } \quad \lim _{\varepsilon \rightarrow 0} \frac{\varepsilon_{j+1}}{\varepsilon_{j}}=0 \quad \text { for } 1 \leq j \leq k-1 .
$$

It should be noted that all results presented here, although stated for naturally separated scales, remain valid for separated scales. The reason for sacrificing generality in this way is that we want to keep technicalities to a minimum. Moreover, with the present application in mind, there is hardly no need to consider other small scales than integer powers of $\varepsilon$. Therefore, throughout this paper, multiscale convergence is defined in the following way:

Definition 3.1. Let $k$ be a positive integer. A bounded sequence $u_{\varepsilon}$ in $L^{p}(\Omega)$ is said to $(k+1)$-scale converge to an element $u$ of $L^{p}\left(\Omega \times \square^{k}\right)$ provided

$$
\lim _{\varepsilon \rightarrow 0} \int_{\Omega} u_{\varepsilon} \phi\left(x, \frac{x}{\varepsilon}, \ldots, \frac{x}{\varepsilon^{k}}\right) d x=\int_{\Omega \times \square^{k}} u \phi d \bar{x}^{k}
$$

for every test function $\phi$ of the form $\phi\left(\bar{x}^{k}\right)=\varphi(x) \prod_{i=1}^{k} \psi_{i}\left(y^{i}\right)$, where $\varphi \in C(\bar{\Omega})$ and $\psi_{1}, \ldots, \psi_{k} \in C_{\mathrm{per}}(\square)$. We write $u_{\varepsilon} \stackrel{k+1}{\rightarrow} u$.

We note that it is possible to replace the set of test functions by $C_{c}^{\infty}\left(\Omega ; C_{\text {per }}^{\infty}\left(\square^{k}\right)\right)$, i.e. the space of all smooth functions $\phi: \Omega \times \mathbb{R}^{k N} \rightarrow \mathbb{R}$ such that $\phi$ is periodic in the second argument and has compact support with respect to $x$.

From the definition of multiscale convergence we see that $(k+1)$ scale convergence implies $k$-scale convergence, 1 -scale convergence being equivalent to weak convergence in $L^{p}(\Omega)$. More precisely, if $u_{\varepsilon} \stackrel{k+1}{\rightarrow} u$, then

$$
u_{\varepsilon} \stackrel{j+1}{\rightarrow} \bar{u}^{k-j}=\int_{\Omega \times \square^{k-j}} u d y^{j+1} \cdots d y^{k} \quad \text { for } 0 \leq j<k .
$$

In particular $u_{\varepsilon} \rightarrow \bar{u}^{k}$ weakly in $L^{p}(\Omega)$.

Definition 3.1 is motivated by the following compactness result: 
Theorem 3.2 (Compactness). Every bounded sequence in $L^{p}(\Omega)$ contains a $(k+1)$-scale convergent subsequence.

Proof. The proof is very similar to the two-scale case, see e.g. ([20], Theorem 7), and is therefore omitted.

The proof of the second important result in multiscale convergence theory, Theorem 3.6 below, is split into several independent results each of which could be interesting in its own right. Before stating these we recall some basic notions and facts that are nonetheless essential to the subsequent analysis.

The $p$-Laplace operator $\Delta_{p}$ is defined for $p \geq 1$ by

$$
\Delta_{p} u=\operatorname{div}\left(|\nabla u|^{p-2} \nabla u\right) .
$$

Recall the well known fact that if $f \in L_{\text {per }}^{p}(\square)$ and $\phi \in L^{q}\left(\mathbb{R}^{N}\right)$ is a measurable function with compact support, then there exists a positive integer $m$ such that

$$
\int_{\mathbb{R}^{N}} f(n x) \phi d x \leq m^{N / p}\|f\|_{L^{p}(\square)}\|\phi\|_{L^{q}\left(\mathbb{R}^{N}\right)} \quad \forall n \in \mathbb{N},
$$

$m$ depending only on the size of the support of $\phi$. The trivial identity

$$
\int_{\square} \psi(n x) d x=\int_{\square} \psi d x \quad \forall \psi \in L_{\text {per }}^{1}(\square), n \in \mathbb{N}
$$

is also useful.

The first result pertains to the image of the div-operator.

Theorem 3.3. For each $f \in W_{\sharp}^{-1, q}(\square)$ there exists a unique $u$ in $W_{\sharp}^{1, p}(\square)$ such that

$$
\langle f, \phi\rangle=\int_{\square}|\nabla u|^{p-2} \nabla u \cdot \nabla \phi d x \quad \forall \phi \in W_{\text {per }}^{1, p}(\square) .
$$

In other words $f=-\operatorname{div} F$, where $F=|\nabla u|^{p-2} \nabla u \in L_{\mathrm{per}}^{q}(\square)^{N}$. Moreover, there exists a constant $C>1$, independent of $f$, such that

$$
\|f\|_{W^{-1, q}(\square)} \leq\left(\int_{\square}|\nabla u|^{p} d x\right)^{1 / q}=\|F\|_{L^{q}(\square)} \leq C\|f\|_{W^{-1, q}(\square)} .
$$

Proof. It is a standard result that the periodic $p$-Poisson equation $-\Delta_{p} u=f$ in $\square$ has a unique solution $u \in W_{\sharp}^{1, p}(\square)$ if and only if $f \in W_{\sharp}^{-1, q}(\square)$. This can be proved either by the direct methods of the 
calculus of variations or by the theory for monotone operators. In terms of weak solutions this establishes (10). By Hölder's inequality

$$
|\langle f, \phi\rangle| \leq\left(\int_{\square}|\nabla u|^{p} d x\right)^{1 / q}\|\phi\|_{W_{\mathrm{per}}^{1, p}(\square)} \quad \forall \phi \in W_{\mathrm{per}}^{1, p}(\square) .
$$

Hence

$$
\|f\|_{W^{-1, q}(\square)} \leq\left(\int_{\square}|\nabla u|^{p} d x\right)^{1 / q} .
$$

Taking $\phi=u$ in (10) and using the Poincaré-Wirtinger inequality yields

$$
\left(\int_{\square}|\nabla u|^{p} d x\right)^{1 / q} \leq C\|f\|_{W^{-1, q}(\square)} .
$$

Next, we apply Theorem 3.3 to a sequence of "almost divergence free" vector fields.

Lemma 3.4. Let $\psi_{1}, \ldots, \psi_{k}$ be functions in $C_{\mathrm{per}}^{1}(\square)$ and let $\Psi$ be a divergence free vector field in $L_{\sharp}^{q}(\square)^{N}$. For $n \in \mathbb{N}$ define

$$
\Psi_{n}^{k}(x)=\prod_{i=1}^{k} \psi_{i}\left(n^{k-i} x\right) \Psi\left(n^{k} x\right) .
$$

Then $\operatorname{div} \Psi_{n}^{k} \in W_{\sharp}^{-1, q}(\square)$ with

$$
\lim _{n \rightarrow \infty}\left\|\operatorname{div} \Psi_{n}^{k}\right\|_{W^{-1, q}(\square)}=0
$$

Proof. Let $u_{n}^{k} \in W_{\sharp}^{1, p}(\square)$ denote the weak solution of $\Delta_{p} u_{n}^{k}=\operatorname{div} \Psi_{n}^{k}$ and set $F_{n}^{k}=\left|\nabla u_{n}^{k}\right|^{p-2} \nabla u_{n}^{k}$. In view of (11), (13) is equivialent to

$$
\lim _{n \rightarrow \infty} \int_{\square}\left|\nabla u_{n}^{k}\right|^{p} d x=0 \quad \text { for } k=1,2, \ldots .
$$

To prove (14) we shall use induction over $k$, but first we need to prove that $\int_{\square}\left|\nabla u_{n}^{k}\right|^{p} d x$ is bounded for each $k$. By definition $u_{n}^{k}$ satisfies

$$
\int_{\square}\left|\nabla u_{n}^{k}\right|^{p} d x=\int_{\square} \prod_{i=1}^{k} \psi_{i}\left(n^{k-i} x\right) \Psi\left(n^{k} x\right) \cdot \nabla u_{n}^{k} d x .
$$


Thus, (11) and Hölder's inequality gives

$$
\left(\int_{\square}\left|\nabla u_{n}^{k}\right|^{p} d x\right)^{1 / q} \leq\|\Psi\|_{L^{q}(\square)} \prod_{i=1}^{k}\left\|\psi_{i}\right\|_{C(\square)} .
$$

Proof of (14) when $k=1$. Using the definition of weak solution we obtain

$$
\int_{\square}\left|\nabla u_{n}^{1}\right|^{p} d x=\int_{\square} \psi_{1} \Psi(n x) \cdot \nabla u_{n}^{1} d x=-\int_{\square} u_{n}^{1} \Psi(n x) \cdot \nabla \psi_{1} d x .
$$

The function $x \mapsto \Psi(n x) \cdot \nabla \psi_{1}(x)$ tends to zero weakly in $L^{q}(\square)$ by the Riemann-Lebesgue lemma. In view of (15) and the Rellich-Kondrachov selection theorem we can extract a subsequence $u_{n_{j}}^{1}$ that converges strongly in $L^{p}(\square)$. The strong and weak convergence implies that $\int_{\square}\left|\nabla u_{n}^{1}\right|^{p} d x \rightarrow 0$ for a subsequence and by uniqueness of the limit it holds for the whole sequence.

Assume now that (14) holds true for $k=\nu$, where $\nu$ is some positive integer. By definition, $u_{n}^{\nu+1}$ satisfies

$$
\begin{aligned}
\int_{\square}\left|\nabla u_{n}^{\nu+1}\right|^{p} d x=\int_{\square} \prod_{i=1}^{\nu+1} \psi_{i}\left(n^{\nu+1-i} x\right) \Psi\left(n^{\nu+1} x\right) \cdot \nabla u_{n}^{\nu+1} d x \\
=\underbrace{\int_{\square} F_{n}^{\nu}(n x) \cdot \nabla\left(\psi_{\nu+1} u_{n}^{\nu+1}\right) d x}_{A_{n}^{\nu}}-\underbrace{\int_{\square} u_{n}^{\nu+1} \Psi_{n}^{\nu}(n x) \cdot \nabla \psi_{\nu+1} d x}_{B_{n}^{\nu}} .
\end{aligned}
$$

Hölder's inequality yields

$$
\left|A_{n}^{\nu}\right| \leq\left(\int_{\square}\left|\nabla u_{n}^{\nu}\right|^{p} d x\right)^{1 / q}\left(\int_{\square}\left|\nabla\left(\psi_{\nu+1} u_{n}^{\nu+1}\right)\right|^{p} d x\right)^{1 / p} .
$$

Because of (15) and the fact that $\int_{\square}\left|\nabla u_{n}^{\nu}\right|^{p} d x \rightarrow 0$ by assumption, it follows that $A_{n}^{\nu} \rightarrow 0$ as $n \rightarrow \infty$. As to $B_{n}^{\nu}$, (15) and the RellichKondrachov selection theorem asserts that we can extract a subsequence $u_{n_{j}}^{\nu}$ that converges strongly in $L^{p}(\square)$. However, the Riemann-Lebesgue lemma and the strong convergence of $u_{n_{j}}^{\nu}$ implies that $B_{n_{j}}^{\nu} \rightarrow 0$. Thus $\int_{\square}\left|\nabla u_{n}^{\nu+1}\right|^{p} d x \rightarrow 0$ for some subsequence and by uniqueness of the limit it holds for the whole sequence. This proves that (14) is true for each $k \geq 1$.

The following statement asserts that vector fields orthogonal to divergence free fields are gradients. 
Theorem 3.5. Suppose $F \in L^{p}\left(\Omega \times \square^{k}\right)^{N}$ satisfies

$$
\int_{\Omega \times \square^{k}} F \cdot \Psi d \bar{x}^{k}=0
$$

for all $\Psi$ in $L^{q}\left(\Omega \times \square^{k}\right)^{N}$ such that $\operatorname{div}_{y^{k}} \Psi=0$. Then $F=\nabla_{y^{k}} u$ for some $u$ in $L^{p}\left(\Omega \times \square^{k-1} ; W_{\sharp}^{1, p}(\square)\right)$.

Proof. Set $V=L^{p}\left(\Omega \times \square^{k}\right)^{N}$. First we project $F$ onto the "space of $y^{k}$-gradients". To this end, define

$$
V_{\text {pot }}=\left\{\nabla_{y^{k}} v: v \in L^{p}\left(\Omega \times \square^{k-1} ; W_{\sharp}^{1, p}(\square)\right)\right\} .
$$

Because of the Poincaré-Wirtinger inequality, $V_{\text {pot }}$ is a closed subspace of $V$. Since $V$ is uniformly convex, there exists a unique element $\nabla_{y^{k}} u$ of $V_{\text {pot }}$ that minimizes the distance from $F$ to $V_{\text {pot }}$, i.e.

$$
\left\|F-\nabla_{y^{k}} u\right\|_{V} \leq\|F-\Phi\|_{V}
$$

for all $\Phi \in V_{\text {pot }}$. Set $\eta=F-\nabla_{y^{k}} u$. Computing the first variation of the minimization problem (17) yields

$$
\int_{\Omega \times \square^{k}}|\eta|^{p-2} \eta \cdot \Phi d \bar{x}^{k}=0
$$

for all $\Phi \in V_{\text {pot }}$. In other words $\operatorname{div}_{y^{k}}|\eta|^{p-2} \eta=0$. Thus we can take $\Psi=|\eta|^{p-2} \eta$ in (16), yielding

$$
0=\int_{\Omega \times \square^{k}} F \cdot|\eta|^{p-2} \eta d \bar{x}^{k}=\int_{\Omega \times \square^{k}}|\eta|^{p} d \bar{x}^{k} .
$$

Hence $\eta=0$, which proves the statement.

The aim of Theorems 3.3, 3.5 and Lemma 3.4 is to prove

Theorem 3.6 (Multiscale limit of gradients). Suppose that $u_{\varepsilon}$ is a sequence in $W_{0}^{1, p}(\Omega)$ such that

(1) $u_{\varepsilon} \rightarrow u$ weakly in $W_{0}^{1, p}(\Omega)$,

(2) $\nabla u_{\varepsilon} \stackrel{k+1}{\rightarrow} \xi \in L^{p}\left(\Omega \times \square^{k}\right)^{N}$.

Then there exists functions $u_{j} \in L^{p}\left(\Omega \times \square^{j-1} ; W_{\sharp}^{1, p}(\square)\right), j=1, \ldots, k$, such that

$$
\xi=\nabla u+\sum_{j=1}^{k} \nabla_{y^{j}} u_{j} .
$$


Proof. To avoid cumbersome notation we write $u_{n}$ in place of $u_{1 / n}$, for all $n \in \mathbb{N}$. Let $\varphi \in C_{c}^{1}(\Omega) ; \psi_{1}, \ldots, \psi_{k-1} \in C_{\text {per }}^{1}(\square)$ and $\Psi \in L_{\text {per }}^{q}(\square)^{N}$ such that $\operatorname{div} \Psi=0$. For $1 \leq j \leq k$, set

$$
I_{n}^{j}=\int_{\Omega} \varphi \Psi_{n}^{j} \cdot \nabla u_{n} d x
$$

where

$$
\Psi_{n}^{j}(x)= \begin{cases}\psi_{1}(n x) \cdots \psi_{j-1}\left(n^{j-1} x\right) \Psi\left(n^{j} x\right) & \text { if } 1<j \leq k \\ \Psi(n x) & \text { if } j=1\end{cases}
$$

The case $j=1$. On the one hand, from the definition of multiscale convergence,

$$
\lim _{n \rightarrow \infty} I_{n}^{1}=\int_{\Omega \times \square} \varphi(x) \Psi\left(y^{1}\right) \cdot \bar{\xi}^{k-1} d \bar{x}^{1} .
$$

On the other hand, using first that $\operatorname{div} \Psi=0$ then the strong convergence of $u_{n}$ and the Riemann-Lebesgue lemma,

$$
I_{n}^{1}=-\int_{\Omega} u_{n} \Psi(n x) \cdot \nabla \varphi d x \rightarrow-\int_{\Omega \times \square} u(x) \Psi\left(y^{1}\right) \cdot \nabla \varphi(x) d \bar{x}^{1}
$$

as $n \rightarrow \infty$. By a density argument, we thus obtain

$$
\int_{\Omega \times \square}\left(\bar{\xi}^{k-1}-\nabla u\right) \cdot \Phi d \bar{x}^{1}=0
$$

for all $\Phi \in L^{q}(\Omega \times \square)^{N}$ satisyfing $\operatorname{div}_{y^{1}} \Phi=0$. Hence, by Theorem 3.5, there exists a $u_{1} \in L^{p}\left(\Omega ; W_{\sharp}^{1, p}(\square)\right)$ such that

$$
\bar{\xi}^{k-1}=\nabla u+\nabla_{y_{1}} u_{1} .
$$

The case $1<j \leq k$. Consider $I_{n}^{j}$ and suppose in addition that $\Psi$ satisfies $\int_{\square} \Psi d x=0$, i.e. $\Psi \in L_{\sharp}^{q}(\square)^{N}$. On the one hand, the definition of multiscale convergence gives

$$
I^{j} \stackrel{\text { def }}{=} \lim _{n \rightarrow \infty} I_{n}^{j}=\int_{\Omega \times \square^{j}} \varphi(x) \psi_{1}\left(y^{1}\right) \cdots \psi_{j-1}\left(y^{j-1}\right) \Psi\left(y^{j}\right) \cdot \bar{\xi}^{k-j} d \bar{x}^{j}
$$

On the other hand, we can write

$$
I_{n}^{j}=\int_{\Omega} \Psi_{n}^{j} \cdot \nabla\left(\varphi u_{n}\right) d x-\int_{\Omega} u_{n} \Psi_{n}^{j} \cdot \nabla \varphi d x .
$$


Estimating the first term yields

$$
\left|\int_{\Omega} \Psi_{n}^{j} \cdot \nabla\left(\varphi u_{n}\right) d x\right| \leq C\left\|\operatorname{div} \Psi_{n}^{j}\right\|_{W^{-1, q}(\square)}\left(\int_{\Omega}\left|\nabla\left(\varphi u_{n}\right)\right|^{p} d x\right)^{1 / p},
$$

where $C$ is a constant depending only on $p$ and $\Omega$. Since $u_{n}$ is bounded in $W_{0}^{1, p}(\Omega)$ and $\left\|\operatorname{div} \Psi_{n}^{j}\right\|_{W^{-1, q}(\square)} \rightarrow 0$ by Lemma 3.4 we conclude that this term tends to zero. As to the second term, the Riemann-Lebesgue lemma and the strong convergence of $u_{n}$ implies that $\lim _{n \rightarrow \infty} \int_{\Omega} u_{n} \Psi_{n}^{j} \cdot \nabla \varphi d x=0$. Thus $I^{j}=0$ and by a density argument this is equivalent to

$$
\int_{\Omega \times \square^{j}}\left(\bar{\xi}^{k-j}-\bar{\xi}^{k-j+1}\right) \cdot \Phi d \bar{x}^{j}=0
$$

for all $\Phi \in L^{q}\left(\Omega \times \square^{j}\right)^{N}$ such that $\operatorname{div}_{y^{j}} \Phi=0$. By Theorem 3.5,

$$
\bar{\xi}^{k-j}-\bar{\xi}^{k-j+1}=\nabla_{y^{j}} u_{j}
$$

for some $u_{j} \in L^{p}\left(\Omega \times \square^{j-1} ; W_{\sharp}^{1, p}(\square)\right)$.

Since $\xi=\bar{\xi}^{0}$ can be written as

$$
\xi=\bar{\xi}^{k-1}+\sum_{j=2}^{k} \bar{\xi}^{k-j}-\bar{\xi}^{k-j+1},
$$

we verify the assertion (18) by applying (19) and (21).

The following theorem could be called the "fundamental theorem of multiscale convergence and monotonicity". A two-scale version of the statement can be found in [17], Theorem 14.

Theorem 3.7 (Multiscale convergence and monotonicity). Let $a: \Omega \times$ $\square^{k} \rightarrow \mathcal{M}_{\alpha, \beta}^{p}(\theta) \subset C\left(\mathbb{R}^{N} ; \mathbb{R}^{N}\right)$ be a Carathéodory function and set

$$
a_{\varepsilon}(x, \xi)=a\left(x, \frac{x}{\varepsilon}, \ldots, \frac{x}{\varepsilon^{k}}, \xi\right) \quad\left(x \in \Omega, \xi \in \mathbb{R}^{N}\right) .
$$

Moreover, let $v_{\varepsilon}$ be a bounded sequence in $L^{p}(\Omega)^{N}$ such that

$$
v_{\varepsilon} \stackrel{k+1}{\rightarrow} v \quad \text { and } \quad a_{\varepsilon}\left(\cdot, v_{\varepsilon}\right) \stackrel{k+1}{\rightarrow} \zeta
$$

for $v \in L^{p}\left(\Omega \times \square^{k}\right)^{N}$ and $\zeta \in L^{q}\left(\Omega \times \square^{k}\right)^{N}$. Then

$$
\liminf _{\varepsilon \rightarrow 0} \int_{\Omega} a_{\varepsilon}\left(x, v_{\varepsilon}\right) \cdot v_{\varepsilon} d x \geq \int_{\Omega \times \square^{k}} \zeta \cdot v d \bar{x}^{k}
$$


and if equality holds, then $\zeta=a(\cdot, v)$.

Proof. Let $\Phi$ be a vector field in $C\left(\bar{\Omega} ; C_{\mathrm{per}}\left(\square^{k}\right)\right)^{N}$ and set

$$
\Phi_{\varepsilon}(x)=\Phi\left(x, \frac{x}{\varepsilon}, \ldots, \frac{x}{\varepsilon^{k}}\right) .
$$

By the monotonicity of $a$

$$
\int_{\Omega} a_{\varepsilon}\left(x, v_{\varepsilon}\right) \cdot v_{\varepsilon} d x \geq \int_{\Omega} a_{\varepsilon}\left(x, v_{\varepsilon}\right) \cdot \Phi_{\varepsilon}+a_{\varepsilon}\left(x, \Phi_{\varepsilon}\right) \cdot\left(v_{\varepsilon}-\Phi_{\varepsilon}\right) d x .
$$

The limit, as $\varepsilon \rightarrow 0$, of the right hand side of the above inequality exists. Hence

$$
\liminf _{\varepsilon \rightarrow 0} \int_{\Omega} a_{\varepsilon}\left(x, v_{\varepsilon}\right) \cdot v_{\varepsilon} d x \geq \int_{\Omega \times \square^{k}} \zeta \cdot \Phi+a\left(\bar{x}^{k}, \Phi\right) \cdot(v-\Phi) d \bar{x}^{k}
$$

and by density and continuity this holds also for any $\Phi$ in $L^{p}\left(\Omega \times \square^{k}\right)^{N}$. Thus, we establish (22) by taking $\Phi=v$.

Suppose now that equality holds in (22). Taking $\Phi=v+t w$ in (23), where $w \in L^{p}\left(\Omega \times \square^{k}\right)^{N}$ and $t \in \mathbb{R}$, we obtain

$$
t \int_{\Omega \times \square^{k}}\left(a\left(\bar{x}^{k}, v+t w\right)-\zeta\right) \cdot w d \bar{x}^{k} \geq 0 .
$$

Dividing by $t$ and using the continuity of $a$ and letting $t \rightarrow 0^{ \pm}$yields

$$
\int_{\Omega \times \square^{k}}\left(a\left(\bar{x}^{k}, v\right)-\zeta\right) \cdot w d \bar{x}^{k}=0
$$

for all $w \in L^{p}\left(\Omega \times \square^{k}\right)^{N}$. Hence $\zeta=a(\cdot, v)$ a.e.

\section{A multiscale homogenization method}

Based on Theorems 3.2, 3.6 and 3.7, we outline a homogenization method for the nonlinear boundary value problem (1).

In view of estimate (8) and the remark following that, the sequence of solutions $u_{\varepsilon}$ to (4) is bounded in $W_{0}^{1, p}(\Omega)$. Applying Theorems 3.2 and 3.6 we can find $u \in W_{0}^{1, p}(\Omega), u_{j} \in L^{p}\left(\Omega \times \square^{j-1} ; W_{\sharp}^{1, p}(\square)\right)(j=1, \ldots, k)$ and $\zeta \in L^{q}\left(\Omega \times \square^{k}\right)^{N}$ such that, up to a subsequence,

(1) $u_{\varepsilon} \rightarrow u$ weakly in $W_{0}^{1, p}(\Omega)$,

(2) $\nabla u_{\varepsilon} \stackrel{k+1}{\rightarrow} \xi=\nabla u+\sum_{j=1}^{k} \nabla_{y^{j}} u_{j}$,

(3) $a_{\varepsilon}\left(\cdot, \nabla u_{\varepsilon}\right) \stackrel{k+1}{\rightarrow} \zeta$. 
Note that

$$
\bar{\xi}^{j}=\nabla u+\sum_{i=1}^{k-j} \nabla_{y^{i}} u_{i} \quad \text { or equivalently } \quad \bar{\xi}^{k-j}=\nabla u+\sum_{i=1}^{j} \nabla_{y^{i}} u_{i} \text {. }
$$

Passing to the limit in the weak formulation (4) and using the defintion of multiscale convergence gives

$$
\int_{\Omega \times \square^{k}} \zeta \cdot \nabla \phi(x) d \bar{x}^{2}=\int_{\Omega \times \square^{k}} b \cdot \nabla \phi(x) d \bar{x}^{k} \quad \forall \phi \in C_{c}^{\infty}(\Omega) .
$$

For $1 \leq j \leq k$, let the testfunction $\phi$ in (4) be

$$
\phi(x)=\varepsilon^{j} \varphi(x) \prod_{i=1}^{j} \psi_{i}\left(\frac{x}{\varepsilon^{i}}\right)
$$

where $\varphi \in C_{c}^{\infty}(\Omega), \psi_{i} \in C_{\mathrm{per}}^{\infty}(\square)(i=1, \ldots, j)$. Then

$$
\begin{aligned}
\int_{\Omega} a_{\varepsilon}\left(x, \nabla u_{\varepsilon}\right) \cdot\left(\varphi \prod_{i=1}^{j-1} \psi_{i}\left(\frac{x}{\varepsilon^{i}}\right) \nabla \psi_{j}\left(\frac{x}{\varepsilon^{j}}\right)+\varepsilon \cdots\right) d x \\
=\int_{\Omega} b_{\varepsilon} \cdot\left(\varphi \prod_{i=1}^{j-1} \psi_{i}\left(\frac{x}{\varepsilon^{i}}\right) \nabla \psi_{j}\left(\frac{x}{\varepsilon^{j}}\right)+\varepsilon \cdot \cdots\right) d x
\end{aligned}
$$

In the limit as $\varepsilon \rightarrow 0$ we obtain

$$
\begin{aligned}
\int_{\Omega \times \square^{k}} \zeta \cdot \varphi(x) \prod_{i=1}^{j-1} \psi_{i}\left(y^{i}\right) \nabla \psi_{j}\left(y^{j}\right) d \bar{x}^{k} & \\
& =\int_{\Omega \times \square^{k}} b \cdot \varphi(x) \prod_{i=1}^{j-1} \psi_{i}\left(y^{i}\right) \nabla \psi_{j}\left(y^{j}\right) d \bar{x}^{k} .
\end{aligned}
$$

By (24), (25) and a density argument it follows that $\zeta$ satisifies

$$
\begin{aligned}
\int_{\Omega \times \square^{k}} \zeta \cdot\left(\nabla \phi(x)+\sum_{j=1}^{k} \nabla_{y^{j}} \phi_{j}\left(\bar{x}^{j}\right)\right) d \bar{x}^{k} & \\
& =\int_{\Omega \times \square^{k}} b \cdot\left(\nabla \phi(x)+\sum_{j=1}^{k} \nabla_{y^{j}} \phi_{j}\left(\bar{x}^{j}\right)\right) d \bar{x}^{k}
\end{aligned}
$$

for all $\phi \in W_{0}^{1, p}(\Omega)$ and $\phi_{j} \in L^{p}\left(\Omega \times \square^{j-1} ; W_{\sharp}^{1, p}(\square)\right)(1 \leq j \leq k)$. 
Let us now characterize $\zeta$. On the one hand, taking $\phi=u$ and $\phi_{j}=u_{j}$ $(1 \leq j \leq k)$ in the identity (26) gives

$$
\int_{\Omega \times \square^{k}} \zeta \cdot \xi d \bar{x}^{k}=\int_{\Omega \times \square^{k}} b \cdot \xi d \bar{x}^{k} .
$$

On the other hand, taking $\phi=u_{\varepsilon}$ in (4) yields

$$
\lim _{\varepsilon \rightarrow 0} \int_{\Omega} a_{\varepsilon}\left(x, \nabla u_{\varepsilon}\right) \cdot \nabla u_{\varepsilon} d x=\lim _{\varepsilon \rightarrow 0} \int_{\Omega} b_{\varepsilon} \cdot \nabla u_{\varepsilon} d x=\int_{\Omega \times \square^{k}} b \cdot \xi d \bar{x}^{k} .
$$

Combining (27) and (28), we see that

$$
\lim _{\varepsilon \rightarrow 0} \int_{\Omega} a_{\varepsilon}\left(x, \nabla u_{\varepsilon}\right) \cdot \nabla u_{\varepsilon} d x=\int_{\Omega \times \square k} \zeta \cdot \xi d \bar{x}^{k}
$$

Appealing to the fundamental theorem of multiscale convergence and monotonicity, Theorem 3.7, we conclude that $\zeta\left(\bar{x}^{k}\right)=a\left(\bar{x}^{k}, \xi\left(\bar{x}^{k}\right)\right)$ for a.e. $\bar{x}^{k} \in \Omega \times \square^{k}$.

The following homogenization result holds.

Theorem 4.1. For $\varepsilon>0$, let $u_{\varepsilon} \in W_{0}^{1, p}(\Omega)$ denote the solution of the variational problem

$$
\int_{\Omega} a_{\varepsilon}\left(x, \nabla u_{\varepsilon}\right) \cdot \nabla \phi d x=\int_{\Omega} b_{\varepsilon} \cdot \nabla \phi d x \quad \forall \phi \in W_{0}^{1, p}(\Omega) .
$$

Then it holds for the whole sequence $u_{\varepsilon}$ that $u_{\varepsilon} \rightarrow u$ weakly in $W_{0}^{1, p}(\Omega)$ and that $\nabla u_{\varepsilon} \stackrel{k+1}{\rightarrow} \xi=\nabla u+\sum_{j=1}^{k} \nabla_{y^{j}} u_{j}$ as $\varepsilon \rightarrow 0$, where $u \in W_{0}^{1, p}(\Omega)$ and $u_{j} \in L^{p}\left(\Omega \times \square^{j-1} ; W_{\sharp}^{1, p}(\square)\right)(1 \leq j \leq k)$. The functions $u$ and $u_{j}$ $(1 \leq j \leq k)$ are the unique functions that satisfy the "homogenized system"

$$
\int_{\Omega \times \square^{k}} a\left(\bar{x}^{k}, \xi\right) \cdot \Phi d \bar{x}^{k}=\int_{\Omega \times \square^{k}} b \cdot \Phi d \bar{x}^{k}
$$

for all $\Phi$ of the form $\Phi\left(\bar{x}^{k}\right)=\nabla \phi(x)+\sum_{j=1}^{k} \nabla_{y^{j}} \phi_{j}\left(\bar{x}^{j}\right)$ where $\phi \in W_{0}^{1, p}(\Omega)$ and $\phi_{j} \in L^{p}\left(\Omega \times \square^{j-1} ; W_{\sharp}^{1, p}(\square)\right)(1 \leq j \leq k)$. Moreover, the following estimate holds

$$
\begin{aligned}
\|\nabla u\|_{L^{p}(\Omega)}^{p} & \leq\left\|\nabla u+\nabla_{y^{1}} u_{1}\right\|_{L^{p}(\Omega \times \square)}^{p} \leq \cdots \\
& \leq\left\|\nabla u+\sum_{j=1}^{k} \nabla_{y^{j}} u_{j}\right\|_{L^{p}\left(\Omega \times \square^{k}\right)}^{p} \leq C\left(1+\|b\|_{L^{q}\left(\Omega ; C\left(\square^{k}\right)\right)}^{q}\right),
\end{aligned}
$$


where $C$ depends only on $p, \beta, \theta$ and $\Omega$.

Proof. We have already proved that a subsequence of $\nabla u_{\varepsilon}(k+1)$-scale converges to some $\xi$ that satisifies (29). However, any $\xi=\nabla u+\sum_{j=1}^{k} \nabla_{y^{j}} u_{j}$ satisfying (29) is unique. For suppose that $\hat{\xi}=\nabla \hat{u}+\sum_{j=1}^{k} \nabla_{y^{j}} \hat{u}_{j}$ is also a solution of (29). Then (29) implies

$$
\int_{\Omega \times \square^{k}}\left(a\left(\bar{x}^{k}, \xi\right)-a\left(\bar{x}^{k}, \hat{\xi}\right)\right) \cdot(\xi-\hat{\xi}) d \bar{x}^{k}=0 .
$$

From the strict monotonicity of $a$ we infer that $\xi=\hat{\xi}$ a.e. Consequently, the whole sequence $\nabla u_{\varepsilon}(k+1)$-scale converges to some $\xi$ as above. Due to the respective assumptions on the functions $u$ and $u_{1}, \ldots, u_{k}$ (being zero on the boundary and having zero integral) we conclude that they are all uniquely determined by $a$ and $b$.

To prove (30) we first note that Jensen's or Hölder's inequality implies

$$
\left\|\bar{\xi}^{k}\right\|_{L^{p}(\Omega)} \leq \cdots \leq\left\|\bar{\xi}^{1}\right\|_{L^{p}\left(\Omega \times \square^{k-1}\right)} \leq\|\xi\|_{L^{p}\left(\Omega \times \square^{k}\right)} .
$$

If $\|\xi\|_{L^{p}\left(\Omega \times \square^{k}\right)}$ is "big" we can find a $C$ such that

$$
\begin{aligned}
C\|\xi\|_{L^{p}\left(\Omega \times \square^{k}\right)}^{p} & \leq \int_{\Omega \times \square^{k}} a\left(\bar{x}^{k}, \xi\right) \cdot \xi d \bar{x}^{k} \\
& =\int_{\Omega \times \square^{k}} b \cdot \xi d \bar{x}^{k} \leq\|b\|_{L^{q}\left(\Omega \times \square^{k}\right)}\|\xi\|_{L^{p}\left(\Omega \times \square^{k}\right)},
\end{aligned}
$$

whence

$$
\|\xi\|_{L^{p}\left(\Omega \times \square^{k}\right)}^{p} \leq C^{-q}\|b\|_{L^{q}\left(\Omega \times \square^{k}\right)}^{q} .
$$

Thus an estimate of the type (30) holds (with a different $C$ ).

\section{Iterated homogenization}

The system (29) is not a suitable form if one is interested in computing the functions $u, u_{1}, \ldots, u_{k}$ of Theorem 4.1. The aim of this section is to devise an iterative algorithm for computing these functions.

To this end, set $\bar{a}^{0}=a$ and for $1 \leq j \leq k$ define $\bar{a}^{j}: \Omega \times \square^{k-j} \rightarrow$ $C\left(\mathbb{R}^{N} ; \mathbb{R}^{N}\right)$ inductively, for $\bar{x}^{k-j} \in \Omega \times \square^{k-j}$ and $\eta \in \mathbb{R}^{N}$, by

$$
\bar{a}^{j}\left(\bar{x}^{k-j}, \eta\right)=\int_{\square} \bar{a}^{j-1}\left(\bar{x}^{k-j}, s, \eta+\nabla v(s)\right) d s,
$$


where $v \in W_{\sharp}^{1, p}(\square)$ is a weak solution of the cell problem

$$
\operatorname{div}_{s} \bar{a}^{j-1}\left(\bar{x}^{k-j}, s, \eta+\nabla v(s)\right)=\operatorname{div}_{s} \bar{b}^{j-1}\left(\bar{x}^{k-j}, s\right) \quad s \in \square .
$$

Remark 5.1. A priori it is not obvious that each $\bar{a}^{j}$ is well defined, let alone continuous and monotone w.r.t. the variable $\eta$. To prove this is somewhat tedious. We therefore do not include the details. What makes things complicated is that the $\bar{a}^{j}$ :s do not map to the same class of continuous, monotone functions as $a$.

Next, (29) implies that for each $1 \leq j \leq k$

$$
\int_{\Omega \times \square^{k}} a\left(\bar{x}^{k}, \xi\right) \cdot \nabla_{y^{j}} \phi_{j}\left(\bar{x}^{j}\right) d \bar{x}^{k}=\int_{\Omega \times \square^{k}} b \cdot \nabla_{y^{j}} \phi_{j}\left(\bar{x}^{j}\right) d \bar{x}^{k} .
$$

for all $\phi_{j} \in L^{p}\left(\Omega \times \square^{j-1} ; W_{\sharp}^{1, p}(\square)\right)$. An equivalent way of stating this is that for a.e. $\bar{x}^{j-1} \in \Omega \times \square^{j-1}, u_{j}$ satisfies

$$
\operatorname{div}_{y^{j}} \int_{\square^{k-j}} a\left(\bar{x}^{k}, \xi\left(\bar{x}^{k}\right)\right) d y^{j+1} \cdots d y^{k}=\operatorname{div}_{y^{j}} \bar{b}^{k-j}\left(\bar{x}^{j}\right), \quad y^{j} \in \square .
$$

We claim that

$$
\int_{\square^{k-j}} a\left(\bar{x}^{k}, \xi\left(\bar{x}^{k}\right)\right) d y^{j+1} \cdots d y^{k}=\bar{a}^{k-j}\left(\bar{x}^{j}, \bar{\xi}^{k-j}\left(\bar{x}^{j}\right)\right)
$$

for $j=0, \ldots, k-1$. The proof is done by induction over $j$. When $j=k-1$ the claim readily follows from (34) and the definition of $\bar{a}^{1}$. Suppose (35) holds true for some $1 \leq j \leq k-1$. For this $j$, (34) can be written

$$
\operatorname{div}_{y^{j}} \bar{a}^{k-j}(\bar{x}^{j}, \underbrace{\bar{\xi}^{k-j+1}\left(\bar{x}^{j-1}\right)+\nabla_{y^{j}} u_{j}\left(\bar{x}^{j}\right)}_{\bar{\xi}^{k-j}\left(\bar{x}^{j}\right)})=\operatorname{div}_{y^{j}} \bar{b}^{j}\left(\bar{x}^{j}\right) \quad y^{j} \in \square .
$$

On comparing (36) to (33), we see that by the definition of $\bar{a}^{k-j+1}$ (see $(32)$ ),

$$
\begin{aligned}
\bar{a}^{k-j+1}\left(\bar{x}^{j-1}, \bar{\xi}^{k-j+1}\left(\bar{x}^{j-1}\right)\right) & =\int_{\square} \bar{a}^{k-j}\left(\bar{x}^{j}, \bar{\xi}^{k-j}\left(\bar{x}^{j}\right)\right) d y^{j} \\
& =\int_{\square k-j+1} a\left(\bar{x}^{k}, \xi\left(\bar{x}^{k}\right)\right) d y^{j} \cdots d y^{k} .
\end{aligned}
$$

Thus (35) is true also for the integer $j-1$ and by induction for all $0 \leq j \leq k-1$. Finally, taking $\phi_{1}=\cdots=\phi_{k}=0$ in (29) and using 
(35) with $j=0$, yields

$$
\operatorname{div}_{x} \bar{a}^{k}(x, \nabla u(x))=\operatorname{div}_{x} \bar{b}^{k}(x) \quad x \in \Omega .
$$

Summing up, we have the following homogenization algorithm:

(1) Solve the cell problem (33) iteratively for $j=1,2, \ldots, k$. At each step compute the function $\bar{a}^{j}$ defined by (32). We call $\bar{a}^{j}$ the "homogenized operator after the $j$ :th iteration".

(2) Solve the homogenized equation (37) to find $u$.

(3) The functions $u_{j}$ are easily found as solutions (that have already been computed) to the cell problems (36).

\section{Application to hydrodynamic lubrication}

The Reynolds equation is a two-dimensional approximation of the pressure that develops in a thin film of viscous fluid (lubricant) as the bounding surfaces are set in relative motion. An important application is in mechanical engineering, where it is used to compute the pressure distribution in various fluid film bearings. A simple form of the Reynolds equation reads

$$
\sum_{i=1}^{2} \frac{\partial}{\partial x_{i}}\left(\frac{h^{3}}{12 \mu} \frac{\partial u}{\partial x_{i}}\right)=\frac{v}{2} \frac{\partial h}{\partial x_{1}} \quad \text { in } \Omega
$$

where $u$ is the unknown pressure distribution, $\Omega \subset \mathbb{R}^{2}$ is the "bearing domain", $h: \Omega \rightarrow \mathbb{R}$ is the film thickness function, $\mu$ is the lubricant viscosity (taken as constant) and $(v, 0)$ is the constant velocity of the lower surface $x_{3}=0$. The upper surface $x_{3}=h\left(x_{1}, x_{2}\right)$ is assumed to remain fixed. To study the influence of surface roughness on several length scales we introduce a small parameter $\varepsilon$ and let the film thickness function be described by the rapidly oscillating function

$$
h_{\varepsilon}(x)=h_{0}(x)+\sum_{j=1}^{k} h_{j}\left(\frac{x}{\varepsilon^{j}}\right)
$$

where $h_{0}: \bar{\Omega} \rightarrow \mathbb{R}$, the smooth film thickness, is continuous and each $h_{j}: \square \rightarrow \mathbb{R}(j=1, \ldots, k)$, the contribution from roughness of wavelength $\varepsilon^{j}$, is continuous and 1-periodic. In addition, we impose suitable restrictions on these functions to ensure that for some $0<\theta<1$ it holds that

$$
\theta \leq h_{\varepsilon}^{3} \leq \theta^{-1} \quad \text { for each } \varepsilon>0 .
$$


The natural boundary condition for the pressure distribution is $u_{\varepsilon}=0$ on $\partial \Omega$. The main homogenization result of this paper, Theorem 4.1, therefore applies by setting

$$
a_{\varepsilon}(x, \xi)=h_{\varepsilon}(x)^{3} \xi \quad b_{\varepsilon}(x)=6 \mu v h_{\varepsilon}(1,0) .
$$

In this case we take $p=\beta=2$ and $\alpha=1$.

The linear structure of (38) is a direct consequence of the the Newtonian properties of the fluid, i.e. a linear relationship between the shear stress and the strain rate. If one assumes a nonlinear constitutive relation for the fluid, it can be shown that the pressure must satisfy a nonlinear equation. Whereas such equations can be considered to fully describe nonNewtonian behaviour, closed form expressions for the terms in the equation, expressed as functions of $h, \nabla u$, are often very complicated or impossible to obtain. Therefore such models have limited value in practice. This leads to the question whether it is possible to modify (38) only slightly so as to capture non-Newtonian effects. In this regard, the following correction has been suggested for incompressible non-Newtonian lubricants which obey the Rabinowitsch constitutive relation, see e.g. He [13] for details,

$$
\sum_{i=1}^{2} \frac{\partial}{\partial x_{i}}\left(\frac{h^{3}}{12 \mu} \frac{\partial u}{\partial x_{i}}+\frac{\kappa h^{5}}{80 \mu}\left(\frac{\partial u}{\partial x_{i}}\right)^{3}\right)=\frac{v}{2} \frac{\partial h}{\partial x_{1}},
$$

where $\kappa>0$ is a constant.

To write (39) in the form (1), choose

$$
a_{\varepsilon}(x, \xi)=h_{\varepsilon}^{3} \xi+\frac{3}{20} \kappa h_{\varepsilon}^{5}\left(\xi_{1}^{3}, \xi_{2}^{3}\right), \quad \text { and } \quad b_{\varepsilon}=6 \mu v h_{\varepsilon}(1,0) .
$$

Theorem 4.1 then applies with $p=\beta=4$ and $\alpha=1$.

To illustrate the utility of homogenization in hydrodynamic lubrication we conclude this section with a concrete example including some numerical simulations. For simplicity we restrict ourselves to roughness on one lengthscale only.

Example 6.1. The geometry of a pocket bearing is shown in Figure 1. It consists of a lower surface $x_{3}=0$ and an upper surface, referred to as a "pad". The pad has a depression, so that a pocket is formed, as well as a small undulation in the $x_{1}$-direction. We wish to analyze the effects of this roughness for very small wavelengths in a non-Newtonian setting by using equation (39). To model this type of bearing, set $\Omega=\left(0, L_{1}\right) \times\left(0, L_{2}\right)$ and $h_{\varepsilon}(x)=h_{0}(x)+A \sin \left(2 \pi x_{1} / \varepsilon\right)$. Here $h_{0}$ is a continuous function which takes the value $h_{\max }$ inside the pocket, the value $h_{\min }$ outside the pocket, except on a set in close proximity to the boundary of the pocket where a smooth 
transition between these two extreme values occurs. Surface and fluid parameters are displayed in Table 1 . These were kept constant throughout a series of simulations which consisted in solving equation (1) with $a_{\varepsilon}$ and $b_{\varepsilon}$ as in (40) for successively smaller values of $\varepsilon$. The result was compared with the homogenized solution. We also computed the homogenized solution for a Newtonian fluid, by setting $\kappa=0$, to compare with the non-Newtonian case.

Conclusions of the experiment. As $\varepsilon \rightarrow 0$ it was observed that the deterministic solutions $u_{\varepsilon}$ converge to the homogenized solution $u$ as illustrated in Figure 3. Moreover, it was seen that the effects of the small surface undulations on the pressure solution is eventually averaged out. Indeed, as seen in Figure 2, the homogenized pressure distribution shows no rapid oscillations. This shows that homogenization is an efficient tool for analyzing the effects of small scale surface roughness. The difference in behaviour between the Newtonian and non-Newtonian fluid is illustrated in Figure 4.

TABLE 1. Input parameters

\begin{tabular}{llll}
\hline Parameter & Description & Value & Unit \\
\hline$L_{1}$ & Pad length $\left(x_{1}\right)$ & 0.1 & $\mathrm{~m}$ \\
$L_{2}$ & Pad width $\left(x_{2}\right)$ & 0.2 & $\mathrm{~m}$ \\
$\mu$ & Fluid viscosity & 0.2 & $\mathrm{~Pa} \mathrm{~s}$ \\
$\kappa$ & Rabinowitsch constant & $5 \cdot 10^{-8}$ & $\mathrm{~Pa}^{-2}$ \\
$A$ & Roughness amplitude & $10^{-6}$ & $\mathrm{~m}$ \\
$h_{\min }$ & Pocket minimum film thickness & $10^{-5}$ & $\mathrm{~m}$ \\
$h_{\max }$ & Pocket maximum film thickness & $1.1 \cdot 10^{-5}$ & $\mathrm{~m}$ \\
$v$ & Speed in $x_{1}$-direction & 1 & $\mathrm{~m} / \mathrm{s}$ \\
\hline
\end{tabular}




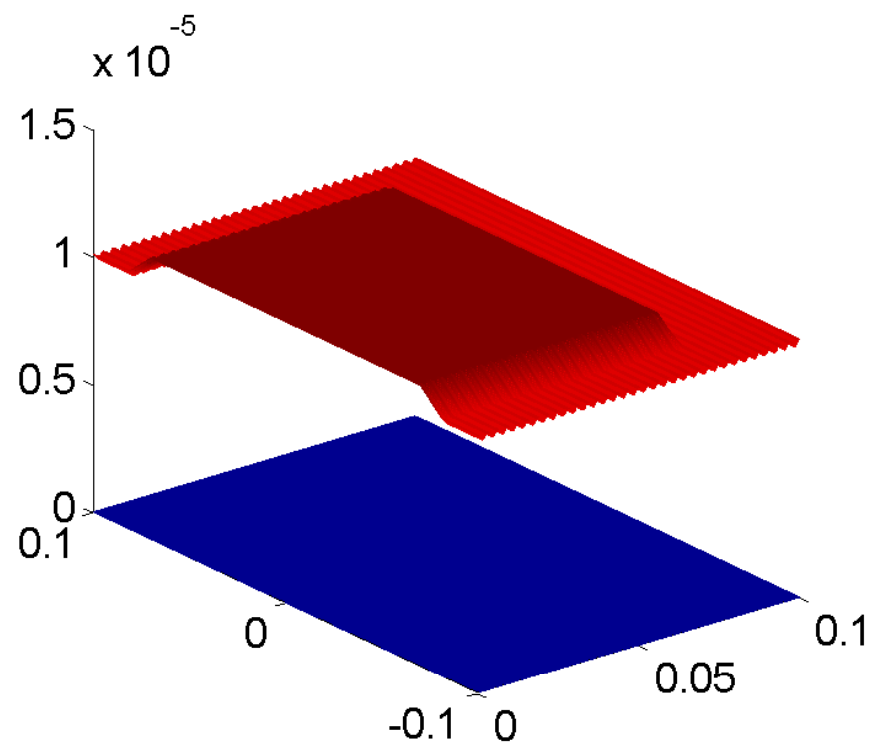

Figure 1. Geometry of a pocket bearing with surface roughness

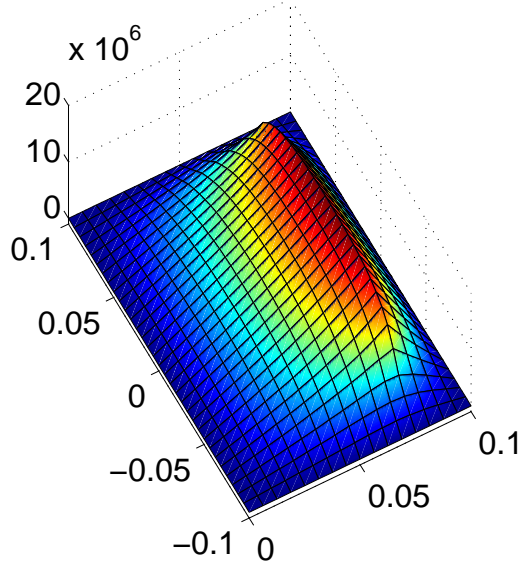

FIGURE 2. Homogenized pressure distribution with a nonNewtonian fluid 


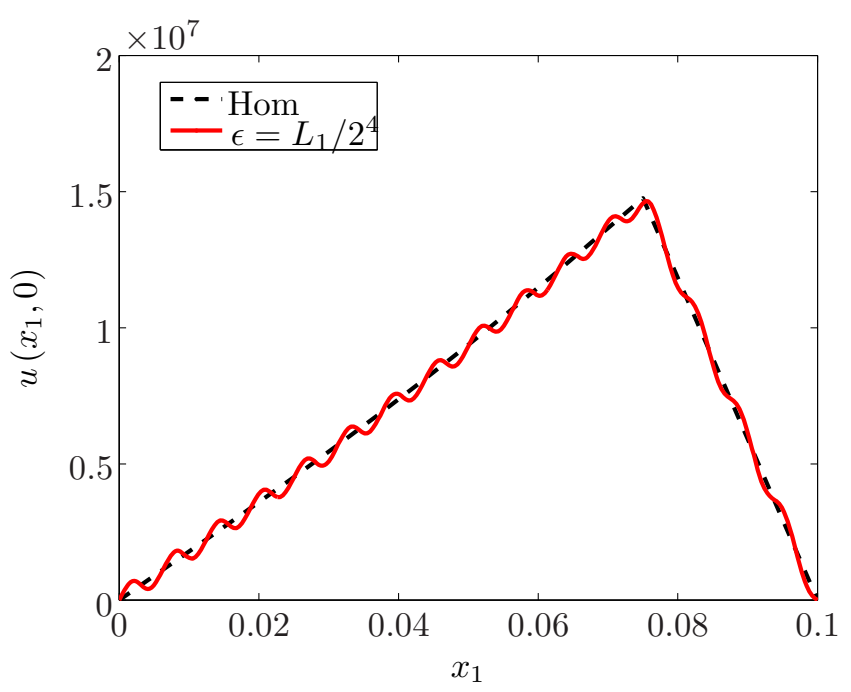

FiguRE 3. Homogenized and deterministic non-Newtonian pressure solutions along the line $x_{2}=0$

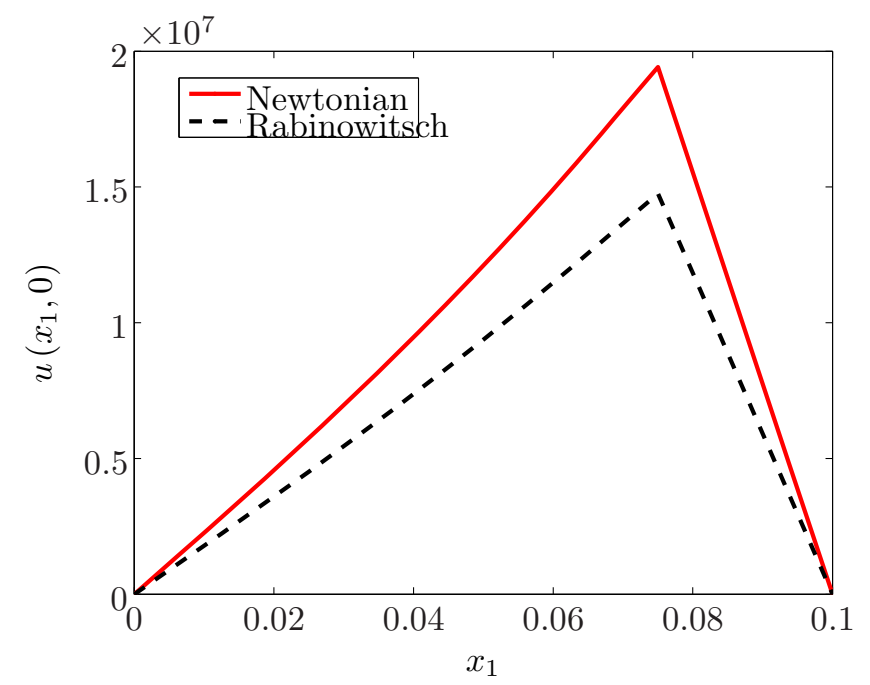

Figure 4. Homogenized Newtonian and non-Newtonian pressure solutions along the line $x_{2}=0$ 
Acknowledgment. The authors wish to thank the anonymous referee for suggestions on improving Section 6 .

\section{References}

[1] G. Allaire, Homogenization and two-scale convergence, SIAM J. Math. Anal., 23 (1992), 1482-1518.

[2] G. Allaire and M. Briane, Multiscale convergence and reiterated homogenization, Proc. R. Soc. Edinburg Sect. A, 126 (1996), 297-342.

[3] E. J. Balder, On compactness results for multi-scale convergence, Proc. Roy. Soc. Edinburgh Sect. A, 129 (1999), 467-476.

[4] A. Almqvist, E. K. Essel, J. Fabricius and P. Wall, Reiterated homogenization applied in hydrodynamic lubrication, Proc. IMechE, Part J: J. Eng. Tribol., 222 (2008), 827-841.

[5] M. Barchiesi, Multiscale homogenization of convex functionals with discontinuous integrand, J. Convex Anal., 14 (2007), 205-226.

[6] G. Bayada and M. Chambat, Homogenization of the Stokes system in a thin film flow with rapidly varying thickness, RAIRO Modél. Math. Anal. Numér., 23 (1989), 205-234.

[7] G. Bayada, S. Martin and C. Vázquez, Two-scale homogenization of a hydrodynamic Elrod-Adams model, Asymptot. Anal., 44 (2005), 75110.

[8] G. Bayada, I. Ciuperca and M. Jai, Homogenized elliptic equations and variational inequalities with oscillating parameters. Application to the study of thin flow behavior with rough surfaces, Nonlinear Anal. Real World Appl., 7 (2006), 950-966.

[9] G. Buscaglia, I. Ciuperca and M. Jai, Homogenization of the transient Reynolds equation, Asymptot. Anal., 32 (2002), 131-152.

[10] I. Ciuperca and M. Jai, Existence, uniqueness and homogenization of the second order slip Reynolds equation, J. Math. Anal. Appl., 286 (2003), 89-106.

[11] A. Bensoussan, J. L. Lions and G. Papanicolaou, Asymptotic Analysis for Periodic Structures, North-Holland, Amsterdam, 1978.

[12] I. Fonseca and E. Zappale, Multiscale relaxation of convex functionals, J. Convex Anal., 10 (2003), 325-350.

[13] J.-H. He, Variational principle for non-Newtonian lubrication: Rabinowitsch fluid model, Appl. Math. Comp., 157 (2004), 281-286.

[14] M. Jai, Homogenization and two-scale convergence of the compressible Reynolds lubrication equation modelling the flying characteristics of 
rough magnetic head over a rough rigid-disk surface, Math. Modelling and Numer. Anal., 29 (1995), 199-233.

[15] J.-L. Lions, D. Lukkassen, L.-E. Persson and P. Wall, Reiterated homogenization of monotone operators, C. R. Acad. Sci. Paris, Sér. I, 330 (2000), 675-680..

[16] J.-L. Lions, D. Lukkassen, L.-E. Persson and P. Wall, Reiterated homogenization of nonlinear monotone operators, Chin. Ann. of Math., 22B (2001), 1-12.

[17] D. Lukkassen and P. Wall, Two-scale convergence with respect to measures and homogenization of monotone operators, J. Funct. Spaces Appl., 3 (2005), 125-161.

[18] D. Lukkassen, A. Meidell and P. Wall, Multiscale homogenization of monotone operators, Discrete and Continuous Dynamical Systems, Ser. A, 22 (2008), 711-727.

[19] G. Nguetseng, A general convergence result for a functional related to the theory of homogenization, SIAM J. Math. Anal., 20 (1989), 608623.

[20] G. Nguetseng, D. Lukkassen and P. Wall, Two-scale convergence, Int. J. Pure Appl. Math., 2 (2002), 35-86.

[21] N. Meunier and J. Van Schaftingen, Periodic reiterated homogenization for elliptic functions, J. Math. Pures Appl., 84 92005), 1716-1743.

[22] P. Wall, Homogenization of Reynolds equation by two-scale convergence, Chin. Ann. of Math., Ser. B 28 (2007), 363-374.

[23] E. Zeidler, Nonlinear Functional Analysis and its Applications II/B, Springer-Verlag, New York Berlin Heidelberg, 1990.

Division of Machine Elements, Luleå University of Technology

SE-971 87 Luleå, Sweden

(E-mail : andreas.almqvist@ltu.se)

Department of Mathematics and Statistics, University of Cape Coast

Cape Coast, Ghana

(E-mail : essemm@ltu.se)

Department of Mathematics, Luleå University of Technology

SE-971 87 Luleå, Sweden

(E-mail : john.fabricius@ltu.se)

Department of Mathematics, Luleå University of Technology

SE-971 87 Luleå, Sweden

(E-mail : wall@sm.luth.se)

(Received : May 2009) 


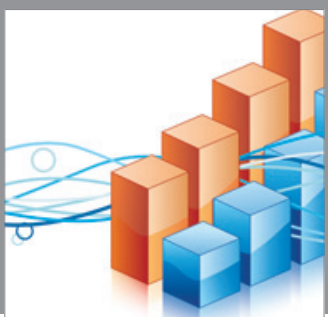

Advances in

Operations Research

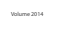

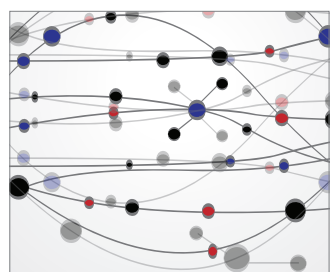

\section{The Scientific} World Journal
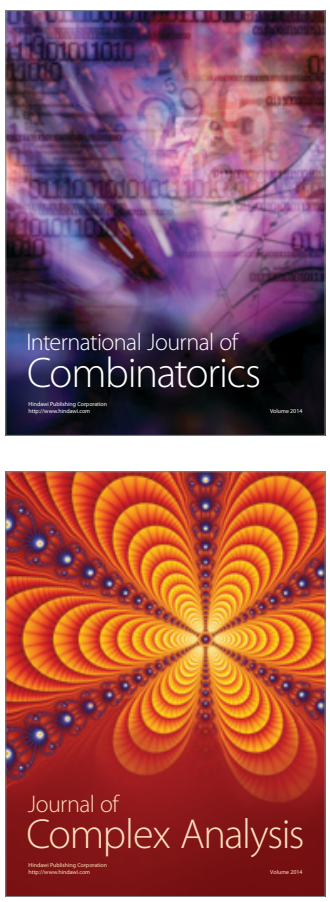

International Journal of

Mathematics and

Mathematical

Sciences
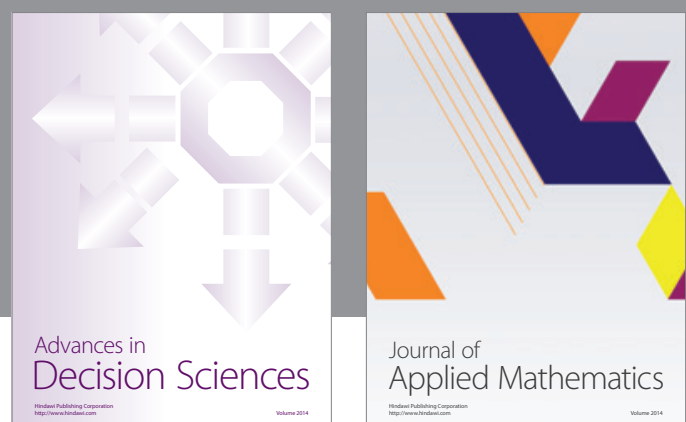

Journal of

Applied Mathematics
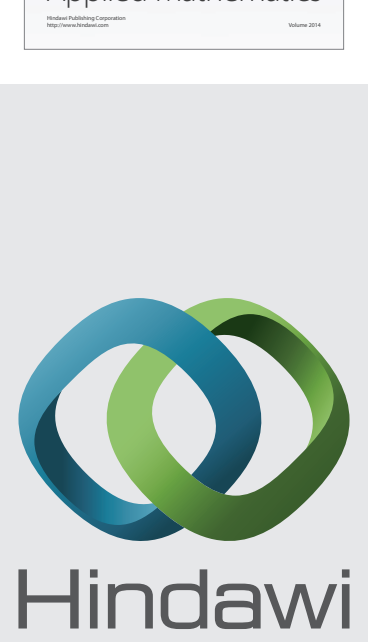

Submit your manuscripts at http://www.hindawi.com
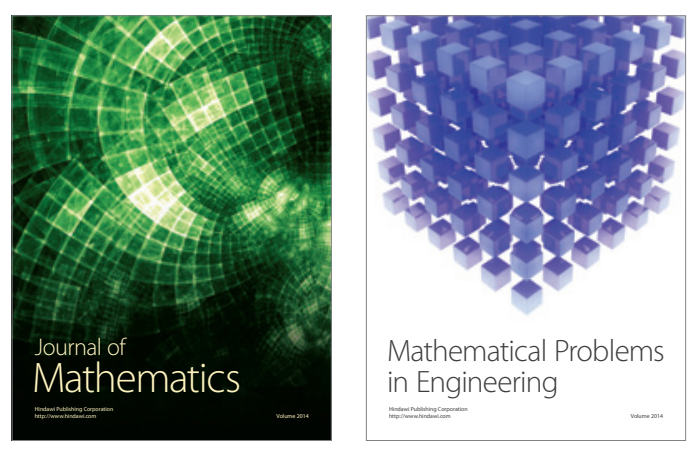

Mathematical Problems in Engineering
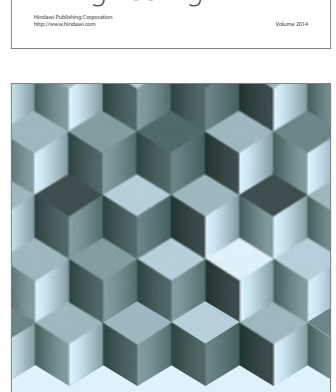

Journal of

Function Spaces
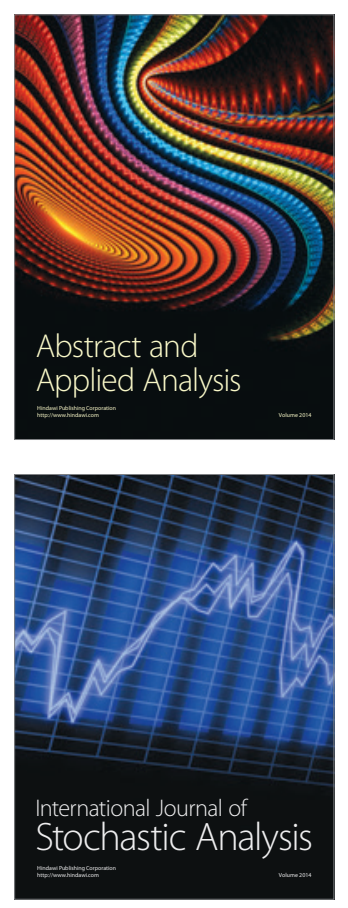

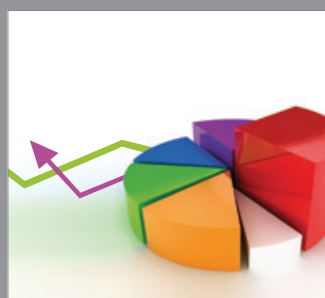

ournal of

Probability and Statistics

Promensencen
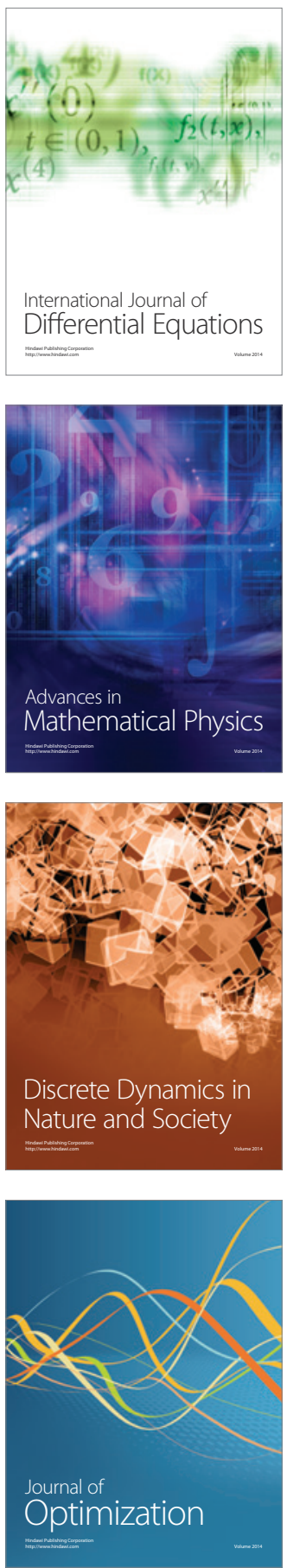